\title{
Ville intelligente, démocratie et durabilité : l'improbable quadrature du cercle
}

\author{
Martin Simard ${ }^{\mathrm{a}}$
}

RÉSUMÉ. La ville intelligente, également appelée smart city, est un concept à la mode qui participe aux stratégies de marketing territorial. Pour une municipalité, le fait de se réclamer de la ville intelligente est un symbole de modernité qui laisse présager l'attractivité, des emplois dans les domaines technologiques et une gestion urbaine plus efficace. Ce virage urbanistique se ferait dans le respect de la démocratie et du développement durable. C'est du moins l'idée qui transpire plus ou moins formellement de la plupart des écrits sur le sujet. Dans cet article, nous adopterons un regard critique sur la ville intelligente en faisant ressortir que les pratiques qui y sont associées pourraient connaître des dérives.

\begin{abstract}
The smart city is a fashionable concept which is involved in many territorial marketing strategies. For a municipality, claiming to be a smart city is a symbol of modernity that involves attractiveness, jobs in technological fields and more efficient urban management. This urban shift would be made with respect for democracy and sustainable development. At least, this is the idea which is more or less formally expressed in most of the writings on the subject. In this paper, we will take a critical look at the smart city concept by pointing out that the practices associated with it could experience drifts.
\end{abstract}

On accole souvent des qualificatifs au mot ville pour illustrer une situation existante ou souhaitée. Ainsi, on utilisera différentes épithètes pour créer des expressions telles que ville du patrimoine, ville nouvelle, ville émergente, ville innovante, ville connectée, voire ville de classe mondiale. D'ailleurs, le concept de ville créative de Richard Florida a amené des échanges animés en milieu universitaire au début des années 2000, malgré sa popularité auprès des administrations locales (Tremblay et Tremblay, 2006) ${ }^{1}$. Dans plusieurs cas, ces expressions contiennent des superlatifs qui relèvent davantage du marketing que de la science (Anttiroiko, 2014). Le concept à la mode de ville intelligente (en anglais, smart city) n'échappe pas à cette règle ${ }^{2}$. Bien au contraire, on peut y voir la quintessence de la modernité urbaine. Du moins, aux dires de ses nombreux promoteurs.

De plus en plus fréquent, le fait de se réclamer de la ville intelligente est un geste symbolique des administrations locales, geste qui laisse présager l'attractivité comme milieu de vie, des emplois dans les domaines technologiques et une gestion urbaine plus efficace. Sur ce dernier plan, on envisage une accessibilité en ligne améliorée des services municipaux, une opération programmée des transports et du traitement des eaux usées, des places et des parcs sécuritaires ainsi qu'une diversité d'applications dans le champ de l'habitation (Dirks et Keeling, 2009). Au-delà des services publics, les secteurs commercial et touristique, parmi tant d'autres, adaptent déjà leurs pratiques afin de satisfaire pleinement une clientèle de plus en plus connectée (publicité ciblée sur la base des données de Google, d'Amazon ou provenant des réseaux sociaux, usage des plateformes Airbnb ou booking.com, etc.). La ville intelligente s'affiche alors comme branchée, aux sens propre et figuré.

Difficile de s'opposer à ce concept des plus flous qui semble intrinsèquement positif. Même si ce dernier a été largement diffusé par le programme Connected Urban Development de Cisco en 2006 et par le concours Smarter Cities Challenge d'IBM en 2008 (Halegoua, 2020)3 , la ville intelligente ne serait pas qu'un outil informatique de grand gabarit, mais aussi une véritable révolution en mesure de régler tous les problèmes des villes, grandes et petites

\footnotetext{
a Professeur, membre du Centre de recherche sur le développement territorial (CRDT), Université du Québec à Chicoutimi
} 
(Gassmann et collab., 2019). Le téléphone intelligent (smartphone) mis sur le marché en 2007 serait son outil de prédilection à travers ses fonctions de communication et de géolocalisation (Courmont et Le Galès, 2019). D’après ses partisans enthousiastes, la ville intelligente s'afficherait inévitablement en tant qu'innovation conviviale, démocratique, durable, économique et sécuritaire.

Devant tant de vertus réelles ou présumées, il n'est pas surprenant de réaliser que la ville intelligente est en voie de devenir le paradigme dominant de l'urbanisme contemporain, en particulier dans les grandes métropoles avides de bien paraitre dans les palmarès de toutes sortes (Eveno, 2018). En conséquence, les cas «exemplaires» se multiplient rapidement sur tous les continents: Amsterdam, Barcelone, Boston, Copenhague, Lyon, Medellín, Rabat, San Francisco, Singapour, Tel-Aviv, Toronto ${ }^{4}$, etc. À elles seules, l'Inde et la Chine comptent des dizaines d'exemples. Des villes de taille moyenne tentent aussi l'expérience (Kansas City, Montpellier, etc.).

Dans cet article, nous poserons un regard critique sur le concept de ville intelligente. L'objectif est de discuter l'assertion explicite ou tenue pour acquise selon laquelle la ville intelligente serait automatiquement démocratique et durable. D'abord, nous tenterons de définir cette notion de même que les champs d'application probables de l'intelligence technologique. Par la suite, nous discuterons des avantages prévus et des dérives possibles, successivement autour des thèmes de la démocratie et de la durabilité. Sur le plan méthodologique, nous procéderons à l'aide d'une analyse de la littérature sur le sujet, à partir de documents institutionnels, scientifiques ou promotionnels.

\section{La ville intelligente : définitions, caractéristiques et débats}

Il importe de définir la ville intelligente avant de poursuivre notre réflexion. Cette expression utilisée ad nauseam par de grandes corporations et différents organismes publics de promotion économique demeure assez peu connue du grand public (Mosco, 2019). Pour la définir, il convient d'abord de mettre en relief le caractère polysémique, voire fluide du concept de ville intelligente ainsi que la diversité des définitions existantes. Un premier auteur souligne les difficultés à bien saisir cet objet :
[La] smart city est l'exemple même d'une notion équivoque et mouvante. Tour à tour modèle, fiction, utopie ou dystopie, elle évolue au gré des objectifs et des contextes d'interventions des acteurs qui se l'approprient. (Khedira, 2020, p. 338)

De manière plus prosaïque, Breux et Diaz (2017) proposent la définition suivante de l'intelligence urbaine en question:

[C’est] une ville pilotée par les données. [...] [Celle-ci] devient une plateforme pour générer des données et des algorithmes. La miniaturisation, la connectivité et l'augmentation de la mémoire flash des technologies digitales [sic] rendent possible lincrustation de dispositifs numériques dans [la gestion des] infrastructures urbaines. (p. 7)

Dans la même veine, Gassmann et ses collègues (2019) suggèrent une définition opérationnelle plus englobante de la ville dite intelligente :

Une ville intelligente applique systématiquement des technologies numériques pour réduire la consommation de ressources, améliorer la qualité de vie de ses habitants et permettre la compétitivité de l'économie régionale de manière soutenue. Celle-ci intègre l'utilisation de solutions intelligentes pour les infrastructures, l'énergie, le logement, la mobilité, les services et la sécurité, solutions qui s'appuient sur des capteurs intégrés, sur la connectivité, sur l'analyse de données et sur des processus à valeur ajoutée indépendants. (p. 25, trad. libre)

En bref, comme le déclarent Languillon-Aussel et ses collègues (2016) :

[C'est] l'ensemble des services urbains [qui] serait concerné par ce processus [d'intelligence], qu'il s'agisse de l'optimisation de la gestion des ressources, des intrants ou des sortants, des déplacements, des relations sociales, voire des citadins eux-mêmes. (p. 36)

Les multinationales de l'informatique arguent que la ville intelligente a pour but de mettre les innovations technologiques au service des administrations publiques et des populations (Dirks et Keeling, 2009). Pour certains observateurs, l'essor, à la fin 
de la décennie 2000-2010, de l'idée de la ville intelligente, et des inévitables technologies qui l'accompagnent, correspond étrangement à la crise économique de 2008 et aux besoins de relance de divers secteurs industriels de pointe (Mosco, 2019). D'ailleurs, il s'agit aussi du moment où l'humanité aurait atteint un taux d'urbanisation de $50 \%$ (UNFPA, 2007), événement historique couramment présenté comme une justification du changement obligé des modes de gouvernance urbaine.

Par-delà les intérêts privés associés au phénomène, il s'impose de comprendre correctement les caractéristiques de la ville intelligente. L'intelligence urbaine serait liée à l'exploitation de gisements de données massives (big data) : «À l'image des matières premières, les données constituent un gisement de ressources exploitables dont l'extraction et la transformation en informations créent de la valeur» (Adam et Rivano, 2020, p. 127). Ces données peuvent être acquises directement par des outils de surveillance et de contrôle (caméras, capteurs, drones, cartes d'accès, etc.) ou achetées à des entreprises informatiques ou autres (en assurance, en pharmaceutique, en sécurité, etc.), organisations qui collectent des données de localisation et de comportements, notamment à partir d'applications de téléphones intelligents ou d'ordinateurs plus traditionnels (voir figure 1). L'internet des objets et l'intelligence artificielle sont aussi parties prenantes de la ville intelligente en permettant aux différents systèmes d'interagir et, éventuellement, de prendre des décisions (Katundi, 2018).

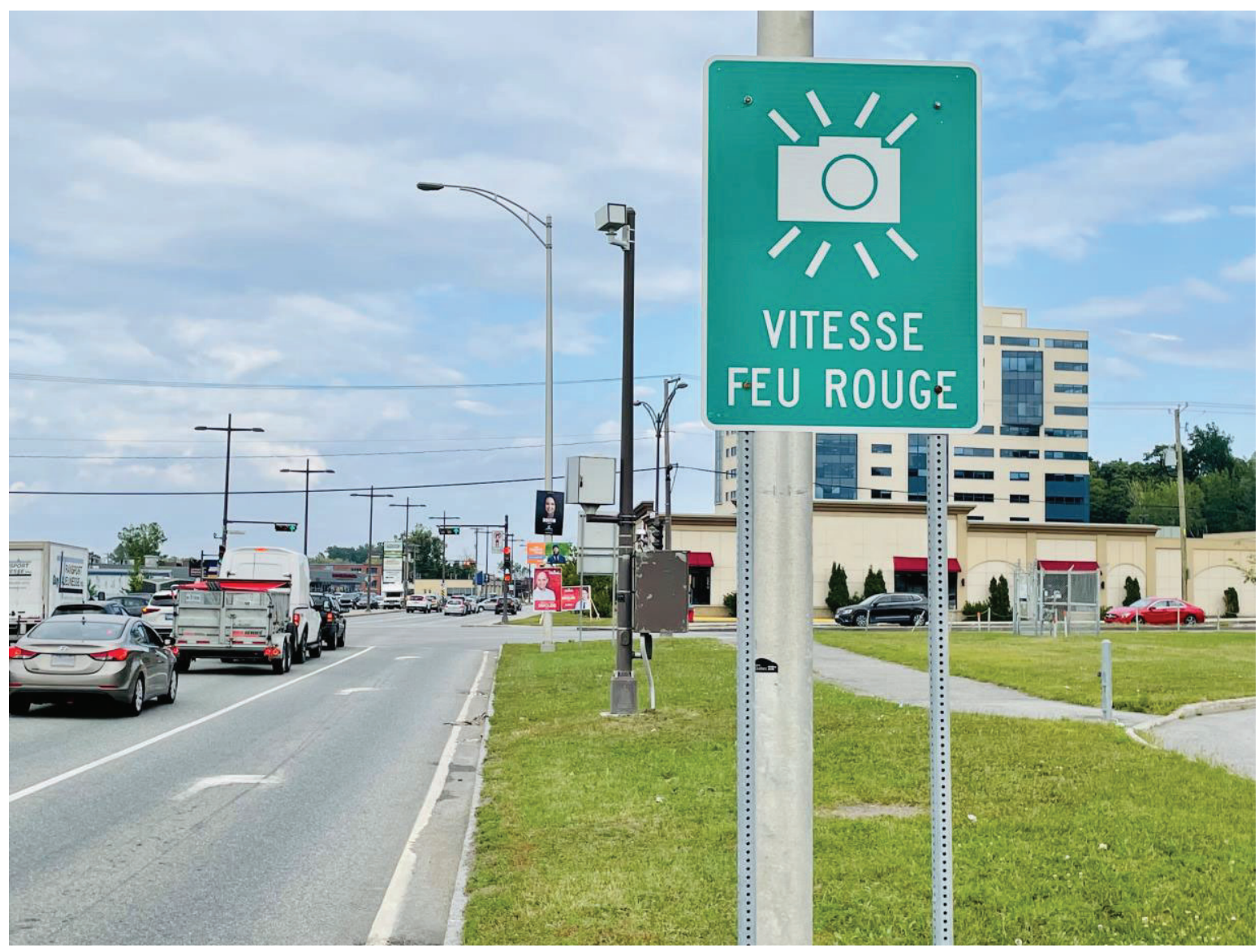

Figure 1 - Le radar photo, un avant-goùt des instruments de la ville intelligente? Source : Martin Simard, 2021 
On peut également concevoir la ville intelligente comme étant le prolongement de la domotique, à une échelle plus vaste. La domotique réfère aux technologies liées aux «bâtiments intelligents». Dans un bâtiment de ce genre, l'éclairage et la température s'ajustent sur commande vocale, les écrans télévisuels sont insérés aux murs, jusqu'au réfrigérateur qui nous indique l'état des denrées alimentaires (Bertuglia, 2020). En fait, la maison de l'avenir est déjà une réalité... à condition de pouvoir y mettre le prix (Merlet, 2018). Cette mention nous rappelle que l'accès à l'intelligence résidentielle et urbaine sera différencié selon les revenus, tant dans le club des métropoles qu'au sein même de chaque ville.

Toujours à propos du bâtiment résidentiel intelligent, celui-ci comporte assurément des avantages, par exemple la possibilité offerte de nous informer à distance de la fréquence et de la configuration des mouvements de personnes handicapées ou en perte d'autonomie (Ben Hadj Mohamed, 2015). Néanmoins, le passage de l'échelle du lot à l'échelle du quartier et de la ville implique des enjeux sociopolitiques, économiques et environnementaux significatifs, lesquels sont le plus souvent ignorés ou sousestimés. En effet, l'achat éventuel d'une maison intelligente par un ménage est un choix personnel qui ne modifie pas globalement la qualité des liens sociaux ni la convivialité des lieux de la vie en société, contrairement à ce qui risque de se produire avec la ville intelligente (Khedira, 2020).

Shearmur et ses collègues (2020) s'intéressent aux enjeux et débats reliés au concept de ville intelligente. Ils soulignent que ce domaine est marqué par un volet de recherche plus technique, notamment sur le thème de l'internet des objets, et par un volet plus social se penchant sur les services aux citoyens. À l'intérieur de ce dernier volet, cinq questions fondamentales émergent fréquemment ${ }^{5}$ :

1. À qui profite le déploiement de la ville intelligente?

2. La croyance selon laquelle la trajectoire technologique actuelle est inéluctable est-elle fondée?

3. Y a-t-il place pour la démocratie dans une ville gérée sur la base d'algorithmes?

4. Le respect de la vie privée est-il possible à l'ère de la marchandisation des données?
5. Peut-on être sûr de la résilience de la ville intelligente face aux changements de toutes sortes?

Dans la même optique, Zuboff (2019) approfondit l'enjeu démocratique en critiquant «le capitalisme de surveillance» en voie d'implantation, dont la ville intelligente serait une émanation. Toutefois, ce changement de société serait un processus qui dépasse les frontières de la ville pour englober l'ensemble des territoires. On parle alors d'intelligence territoriale (Pelissier et Pybourdin, 2009). Si l'on tient compte du fait que $81 \%$ des Québécois possèdent maintenant un téléphone intelligent (ATN, 2020), la possibilité de localiser les gens en tout temps ouvre la porte à des formes de surveillance (monitoring) diverses. D'ailleurs, plusieurs compagnies mettent à profit ces possibilités depuis des années, ce qui permet notamment de nous informer sur la densité de la circulation automobile ou de nous offrir de la publicité «localisée». Au-delà de ces usages pratiques, l'enregistrement et l'archivage de nos "préférences» font surgir de profonds questionnements d'ordres éthique, politique et philosophique.

\section{Les villes intelligentes à l'épreuve de la démocratie}

La nature même de la démocratie serait interpellée par l'émergence de la ville intelligente (Gagné, 2019). En effet, la simple informatisation de l'acte de voter laisserait sur la touche les électeurs illettrés en matière de technologies. De plus, les algorithmes présents sur les réseaux sociaux, comme Facebook ou Instagram, auraient déjà contribué à l'appauvrissement des débats démocratiques en période électorale ou non en relayant aux usagers, c'est-à-dire un très fort pourcentage des citoyens, des messages les réconfortant dans leurs «préférences» (Proulx, 2021). Ce phénomène inattendu favoriserait tout autant la manipulation des électeurs que la polarisation du vote à tous les paliers gouvernementaux, dont l'échelon local ou métropolitain. De surcroît, une fois les élections passées, l'imputabilité des actions politiques aura sans doute tendance à migrer de l'élu(e) vers la machine.

De manière plus criante, la multiplicité des données utilisées dans la gestion des mécanismes régulateurs de la ville pose ou posera de manière frontale la question du respect de la vie privée des citoyens (Courmont et Le Galès, 2019). Ces données massives 
sont croisées, géolocalisées, associées à des «utilisateurs », ce qui ouvre la porte à de multiples usages, pas toujours d'intérêt public... S'il faut en croire la fameuse loi de Murphy ${ }^{6}$, on peut aisément prévoir un usage policier des données dans un futur plus ou moins rapproché. Au demeurant, les algorithmes de l'avenir connaittront probablement mieux que nousmêmes nos besoins et désirs, nous dégageant de responsabilités de faire des choix (repas, loisirs, itinéraires, sélection d'un conjoint, etc.), ce qui est à la base même de notre identité (Harari, 2018).

Qu'à cela ne tienne, divers auteurs ou intervenants mettent l'accent sur les dimensions démocratique, participative ou durable de la ville intelligente (Meijer et collab., 2016). D'ailleurs, des agences officielles, par exemple l'administration du programme Habitat de l'ONU, la Commission européenne ou l'Union des municipalités du Québec ${ }^{7}$, supportent et promeuvent le concept de ville intelligente sur la base d'une vision optimiste de ce projet d'envergure internationale, réalisé de façon différenciée au palier local (Courmont et Vincent, 2020). Sur le terrain, l'intérêt des agences d'urbanisme peut varier d'un endroit à l'autre (Vidal et Gauthier, 2021). Certains analystes identifient aussi des modèles de villes intelligentes qui protègent la souveraineté locale et le «droit à la ville »" à l'exemple de Barcelone (Durand Folco, 2019)9. Néanmoins, on doit se demander si ces adhésions, qui s'appuient sur des conceptions généreuses, sont appropriées puisque la ville intelligente peut favoriser des pratiques de surveillance et de contrôle des citoyens, sans compter la vulnérabilité de tout «l'appareil» aux catastrophes économiques, sanitaires, naturelles ou aux simples pannes d'électricité.

En outre, on peine à comprendre comment les technologies pourraient aider à combattre des problèmes récurrents comme la pauvreté, les inégalités et l'accès au logement ou les quartiers non viabilisés dans les pays du Sud, c'est-à-dire les bidonvilles ou favelas. L'Inde, qui compte une forte population urbaine pauvre et sans services de base, s'est engagée dans un programme ambitieux de villes intelligentes «qui porte une violence symbolique [et] sonne comme une provocation » (Adam et Rivano, 2020, p. 10). La mise à l'écart de ces problèmes urbains fondamentaux pour se lancer corps et âme sur la voie des technologies ressemble fort à une fuite en avant. Cette fuite, dont je me garderai de faire l'éloge ${ }^{10}$, ne laisse présager rien de bon pour l'avenir de notre monde fortement urbanisé.
Sur le plan philosophique, rappelons que l'association automatique entre progrès technique et progrès social est largement inscrite dans l'histoire et la psyché de l'Occident, depuis la révolution industrielle (Vaccari, 1957). La résilience de cette croyance n'en fait pas une réalité pour autant. Nonobstant cela, la ville intelligente s'inscrit clairement dans cette tendance séculaire à la fétichisation de la technique, «où toutes les questions et réponses débutent et se terminent par des solutions technologiques» (Mosco, 2019, p. 1, trad. libre). D'ailleurs, ces villes intelligentes pourraient facilement devenir le paradis des pirates informatiques (hackers), une caste de lettrés ayant son propre «programme» en cette période pas si lointaine de numérisation extrême. La ville intelligente, pourtant promue comme résiliente, ne serait alors pas exempte de vulnérabilité.

Sur le terrain, les dérives de la ville intelligente sont déjà visibles dans plusieurs métropoles chinoises. On y retrouve des millions de capteurs dotés de capacités de reconnaissance faciale (Briner, 2020). Un système de pointage est aussi établi pour mesurer la rectitude des comportements des populations sur la base de technologies de localisation (Henriot, 2018). L'Inde expérimente également un système de comptabilité citoyenne à l'aide de la carte d'identité numérique Aadhaar (Beckouch, 2019). À partir de ces exemples troublants, on peut aisément imaginer l'avènement prochain d'une société orwellienne ou foucaldienne, où le citoyen n'est plus qu'une marionnette et au sein de laquelle les élus locaux seront probablement sélectionnés à l'aide d'algorithmes. Bien entendu, l'Occident n'est pas à l'abri de telles dérives, même si celles-ci pourraient prendre des formes plus subtiles s'appuyant sur la «servitude volontaire» (Lévesque, 2019) d'une large cohorte de citoyens-consommateurs en liberté surveillée (Damon, 2016) ${ }^{11}$.

\section{Les villes intelligentes à l'épreuve de la durabilité}

Le concept de ville intelligente s'entrecroise fréquemment avec une autre expression à la mode: la ville durable. La combinaison des deux notions devient alors le nec plus ultra en urbanisme ou en administration publique (Boutros, 2019). Plus encore, pour un grand nombre de chercheurs et d'acteurs sur le terrain, la ville intelligente ne peut être que durable. Même s'il est possible d'obtenir des gains substantiels dans l'émission de GES grâce à la coordination des 
feux de circulation, par l'entremise d'un éclairage urbain mieux ciblé et par une programmation rigoureuse du traitement et du recyclage des déchets à l'aide des technologies de pointe (McKenna, 2021), cette combinaison ne va pas de soi, quoiqu'on puisse évidemment militer et travailler en sa faveur (Morozov et Bria, 2018).

De manière générale, on peut soutenir que le caractère durable de la ville intelligente n'est guère garanti. Par exemple, les réductions d'utilisation de carburants fossiles ou d'énergies d'origines diverses, qu'on estime à approximativement 20\% (Haëntjens, 2018), risquent d'être compensées par la consommation en ressources des centres de traitement de données (voir tableau 1). Ces immenses bâtiments sans âme contenant généralement peu d'employés se multiplient actuellement au Québec (Halin, 2017) ${ }^{12}$. On en compte plus d'une cinquantaine, concentrés surtout dans la grande région métropolitaine de Montréal et, dans une moindre mesure, à Québec (voir figure 2). D’ailleurs, le principe du «plus de données, plus de chaleur » pourrait signifier une demande croissante en énergie (Perreault, 2019). Toutefois, les lieux de stockage des données sont délocalisés. Ils dépendent très peu des pratiques locales en matière d'intelligence urbaine.

\begin{tabular}{|c|c|c|}
\hline Date & Localisation & Gestionnaire de centre de données \\
\hline 2011 & Montréal & TATA (TCS) \\
\hline 2012 & Rimouski & TELUS \\
\hline 2012 & Gatineau & Bell \\
\hline 2013 & Montréal & $\mathrm{OVH}$ \\
\hline 2013 & Montréal & Leaseweb Global (anciennement INAP iWeb) \\
\hline 2014 & Québec & Vantage (anciennement Vidéotron/4 Degrés) \\
\hline 2015 & Kirkland & Cogeco \\
\hline 2015 & Longueuil & COLO-D \\
\hline 2015 & Québec & Microsoft \\
\hline 2015 & Drummondville & IBM \\
\hline 2016 & Baie-D’Urfé & ROOT \\
\hline 2016 & Vaudreuil-Dorion & GI Partners (anciennement Ericsson) \\
\hline 2016 & Pointe-Claire & Vantage (anciennement Hypertech) \\
\hline 2017 & Montréal & Urbacon \\
\hline 2017 & Montréal & Vantage (anciennement Vidéotron/4 Degrés) \\
\hline 2018 & Montréal & Google \\
\hline 2019 & Varennes & Amazon \\
\hline Projet & St-Bruno-de-Montarville & Vantage \\
\hline Projet & Beauharnois & Google \\
\hline Projet & Lévis & QScale \\
\hline
\end{tabular}

Tableau 1 - Les 20 principaux centres de données infonuagiques par date d'implantation au Québec Source : Investissement Québec, 2021 ${ }^{13}$; La Presse, 2020 ${ }^{14}$; Journal de Québec, $2017^{15}$

Il faut prendre conscience du fait que les centres de données infonuagiques doivent mobiliser beaucoup d'électricité pour climatiser des équipements informatiques massifs en constante activité ${ }^{16}$. On estime que «l'empreinte carbone des centres de données est comparable à celle du transport aérien, [soit] $2 \%$ des émissions de gaz à effet de serre totales » (Joarson,
2021, p. 1). L'eau est de plus en plus mise à contribution pour rafraîchir ces complexes, mais cette pratique n'est pas véritablement meilleure d'un point de vue environnemental. Bref, on oublie souvent qu'Internet et l'infonuage (cloud) comportent des ancrages physiques nombreux et qu'ils ne sont pas sans impacts sur l'aménagement du territoire et sur les écosystèmes naturels (Cattaruzza, 2019). 


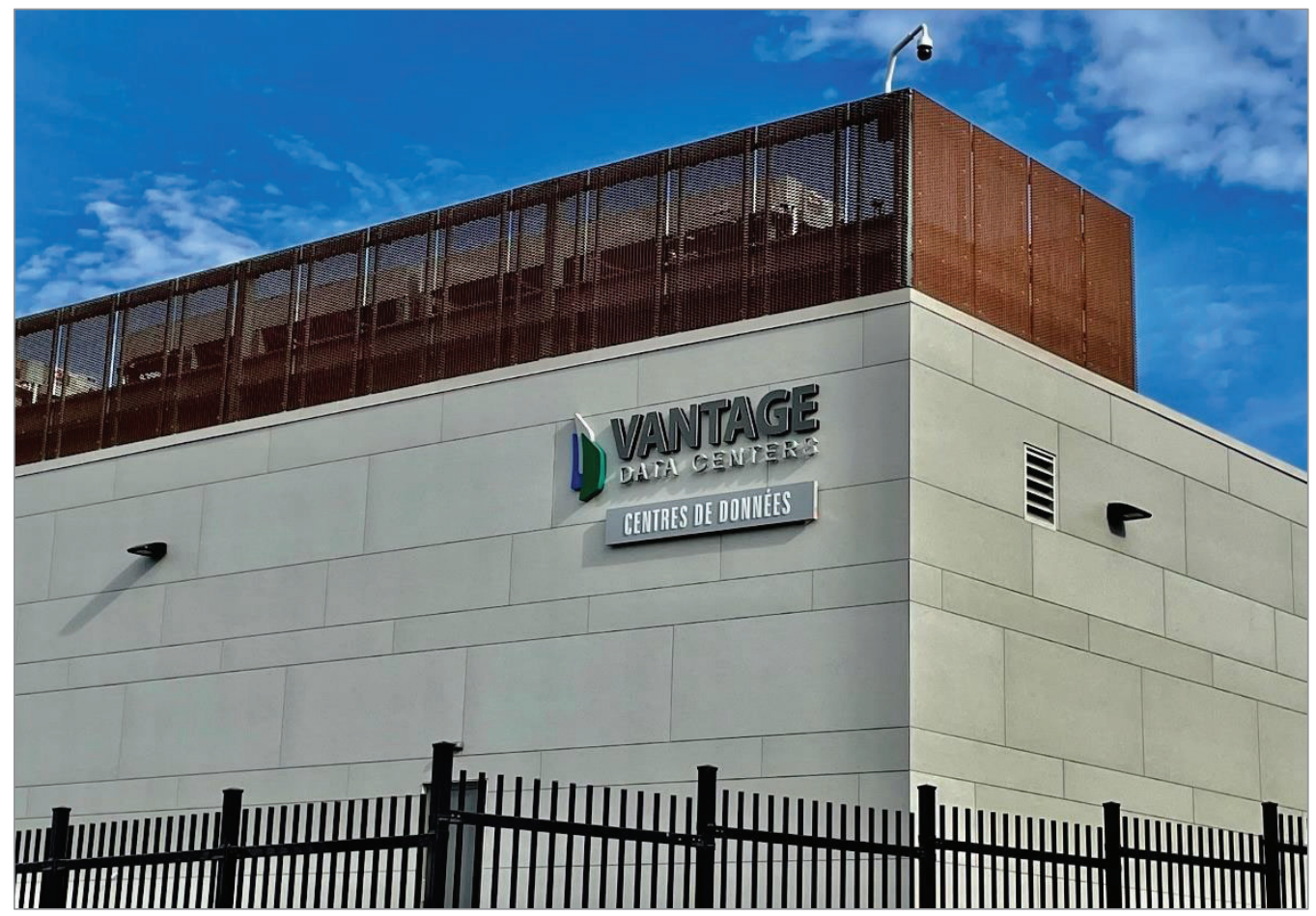

Figure 2 - Le centre de données Vantage dans le Parc technologique du Québec métropolitain Source : Martin Simard, 2021

Une autre critique concernant les prétentions de la ville intelligente en regard du développement durable concerne les changements climatiques. L'ampleur des changements à venir (inondations, vagues de chaleur, incendies, etc.) et leurs effets sont généralement peu traités par la littérature sur la ville intelligente. À cet égard, Mosco (2019) affirme que «les promoteurs des villes intelligentes ont tendance à ignorer les changements climatiques ou ils proposent de les contrecarrer par des solutions technologiques aux effets surestimés » (p. 8, trad. libre). D’ailleurs, on se demande bien comment des équipements informatiques pourraient combattre des désastres naturels majeurs, à part prévenir les occurrences par l'intermédiaire de la surveillance d'indicateurs et lancer des alertes en cas de sinistre imminent.

Au surplus, la valorisation des solutions technologiques aux divers problèmes de société et le délestage d'activités de gestion aux algorithmes ou instruments de l'intelligence artificielle pourraient bien contrer la nécessaire mobilisation en faveur de la durabilité en ville, par le biais d'une perte d'autonomie citoyenne (Paquot, 2020). Aurons-nous la motivation pour mettre en place un jardin communautaire ou pour nous liguer contre l'abattage d'arbres par les autorités, alors qu'au quotidien les luminaires s'éteignent par un simple mot et qu'un texto des plus brefs permet l'arrivée rapide d'un transport Uber ou la livraison sans délai de goûters en tout genre? Une apathie générale pourrait bien caractériser les résidents de la ville intelligente (Gagné, 2019) ainsi qu'une perte du sens du lieu (Quesnot, 2016), et limiter tant son caractère démocratique que la capacité des citoyens à mettre en place des projets de développement durable ou de nature communautaire. Les transformations du lien social et du lien spatial ne constituent pas de minces affaires.

Finalement, cette probable apathie citoyenne, qui pourrait se développer et restreindre les actions spontanées ou organisées de développement durable participatif, opérera en parallèle avec l'augmentation des moyens pour mettre en place une durabilité urbaine de type coercitif. Il va de soi que divers instruments de mesure des taux de pollution par secteur géographique ou de supervision des niveaux de consommation d'eau ou d'énergie par bâtiment ou par ménage seront disponibles et aisés à faire fonctionner dans un avenir rapproché. La CityScore de Boston joue déjà ce rôle de surveillance de l'état de santé globale de la ville et de ses quartiers depuis quelques années (Beckouch, 2019). Si la durabilité trouve sa place dans la ville intelligente, ce sera donc sous sa forme la moins souhaitable, 
soit un genre d'environnementalisme autoritaire qui ne correspond pas réellement à la définition du développement durable (Theys, 2020).

\section{Conclusion}

La ville intelligente mérite davantage d'attention avant d'en faire la promotion et de la mettre en œuvre. À côté des défis technologiques, qui peuvent être emballants pour les spécialistes de différents domaines, de multiples enjeux sociaux, politiques et environnementaux se profilent à l'horizon. Il est vrai que plusieurs de ces enjeux concernent la société du numérique dans son ensemble, et non seulement le concept de ville intelligente. Néanmoins, la ville, en particulier les métropoles, constitue un lieu de concentration des populations, des infrastructures, des institutions, des sièges sociaux corporatifs, des activités de recherche, etc. Elle est donc un territoire « étroit et unique » (Tribillon, 2009, p. 5) des plus propices au développement, à l'expérimentation et à l'implantation de technologies intelligentes, donc au surgissement des effets positifs et néfastes de l'exploitation de ces techniques de pointe.

L'utilisation de procédés technologiques dans des domaines comme la gestion des transports publics ou l'accès aux stationnements ne doit pas être perçue comme problématique ni à proscrire. Ce n'est pas le point de vue entretenu dans cet essai critique sur ce sujet. Il en va de même pour les projets de grappes industrielles technologiques. Le problème de la ville intelligente réside plutôt dans la volonté affirmée d'arrimer tous ces différents systèmes gérés par des ordinateurs et des algorithmes ainsi que d'utiliser ces technologies dans la gouvernance même de la cité. Tel que nous l'avons mentionné, de nombreux enjeux ressortent de la perspective globalisante de l'intelligence territoriale véhiculée par l'idée de ville intelligente.

Les appels à la prudence ont malheureusement peu de chance d'être entendus. Pourquoi revenir constamment sur les problèmes persistant de pauvreté ou d'accès au logement, alors qu'il est plus commode et stimulant de tourner notre attention vers de nouvelles idées et de nouveaux outils d'intervention? La ville intelligente offre ce déplacement de l'objectif qui répond visiblement aux besoins psychologiques de nouveauté chez l'humain, tant sur le plan vestimentaire ou alimentaire qu'en administration publique, en urbanisme ou en sciences. En tant que nouveau principe à la mode, l'intelligence urbaine promet en prime de régler tous les problèmes, les anciens comme les nouveaux. Pourquoi diable résister au chant des sirènes puisque tous y trouvent leur compte... sauf les plus démunis?

Lancées simultanément, les invitations à la «simplicité volontaire» ou à la «frugalité heureuse $»^{17}$ ne sont guère populaires, même si elles conviennent davantage à l'équilibre écologique d'une planète approchant les 8 milliards d'individus. Quant à la démocratie, la montée de l'autoritarisme (Chine, Russie, Turquie, etc.) et du populisme à tendance fasciste (Brésil, États-Unis, Hongrie, Pologne, etc.), combinée au maintien de la dictature ou de démocraties de façade dans plusieurs pays (Algérie, Arabie saoudite, Égypte, Myanmar, etc.), laisse présager qu'il aura s'agit d'une période historique assez brève, au final. Heureusement, la cybergouvernance nous laissera tout le temps libre pour vaquer à des activités programmées et supervisées...

\section{NOTES}

1 La thèse de la ville créative est controversée, car elle soutient que le développement des grandes villes et métropoles est lié à l'attraction de populations dites créatives. D'une part, cela suppose une compétition entre les villes dans un jeu à somme nulle à l'échelle nationale. D'autre part, il y a débat sur quels segments de la population doivent être considérés créatifs ou non créatifs, sans compter le caractère élitiste d'un tel classement.

2 La traduction de smart city par ville intelligente fait d'ailleurs l'objet de débats et de discussions, comme l'explique Goin (2017).

3 Cette auteure fait remonter l'origine de la ville intelligente beaucoup plus loin dans le temps. Elle mentionne l'influence probable de l'exposition Futurama de New York en 1939 (idée de l'automatisation de la circulation automobile) ou les travaux de Jay Forrester sur la « dynamique urbaine », qui datent de 1969 (idée de la ville comme système modélisable).

4 Le projet du quartier intelligent modèle de Quayside à Toronto a soulevé de multiples controverses. Celui-ci a été abandonné en mai 2020 (https:/ /onezero.medium.com/how-a-band-of-activists-and-one-tech-billionaire-beat-alphabets-smart-cityde19afb5d69e). 
5 Ces chercheurs identifient en fait six questions « de type social » associées à la ville intelligente. Nous en avons regroupé ici deux qui portent sur la démocratie afin d'alléger le texte.

6 La loi de Murphy est un principe non scientifique énoncé par Edward Murphy, un ingénieur en aéronautique américain. Cet adage, popularisé par les médias à la fin des années 1940, propose que «si quelque chose peut mal tourner [dans une expérience scientifique ou dans la vie en société], alors cette chose finira infailliblement par mal tourner » (https://www.techno-science.net).

7 https://umq.qc.ca/dossiers/amenagement-et-urbanisme/villes-intelligentes

8 Nous reprenons ici la formule célèbre et encore fréquemment employée du sociologue français Henri Lefebvre.

9 La Déclaration de Montréal pour un développement responsable de l'intelligence artificielle de 2017 illustre une volonté d'aller dans cette direction (https://www.declarationmontreal-iaresponsable.com/la-declaration).

10 Nous référons ici à l'ouvrage L'éloge de la fuite d'Henri Laborit (1976). La fuite y est présentée comme un comportement adéquat dans certains contextes.

11 Il faut noter que Damon énonce deux visions de la ville intelligente, l'une plus orwellienne, l'autre plus conviviale. Nous référons ici à la première option.

12 Le Québec constitue une importante zone de concentration de centres de données, même s’il demeure moins important que l'État américain de la Virginie ou la région de l'île-de-France, à cet égard (https:/ /www.lesoleil.com/2021/10/27/le-quebec-seveut-le-nouvel-eldorado-vert-et-pas-cher-des-centres-de-donnees-8fec53e9104540aad7dcb20b5427e151)

13 https://www.investquebec.com/international/fr/secteurs-activite-economique/technologies-informationcommunications/centres-de-donnees.html

14 https://www.lapresse.ca/affaires/techno/2020-11-21/centre-de-donnees/achat-et-agrandissement-pour-vantage-canada.php

15 https://www.journaldemontreal.com/2017/07/16/le-quebec-est-un-paradis--des-centres-de-donnees

16 Au Québec, l'utilisation de l'hydroélectricité réduit les effets de cette forte consommation énergétique, mais les centres de données américains utilisent souvent de l'énergie polluante produite par des centrales au charbon.

17 Ces expressions sont successivement de Serge Mongeau (2005) et de Thierry Paquot (2020).

\section{RÉFÉRENCES}

Académie de la transformation numérique (ATN). (2020). NETendances 2020 : portrait numérique des foyers québécois, 11(3). https://transformation-numerique.ulaval.ca/wp-content/uploads/2020/12/netendances-2020-portrait-numerique-des-foyersquebecois.pdf

Adam, M. et Rivano, H. (2020). Données : quand le numérique produit et gouverne la ville. Dans M. Adam et É. Comby (dir.), Le capital dans la cité : une encyclopédie critique de la ville (p. 125-137). Éditions Amsterdam.

Anttiroiko, A.-V. (2014). The political economy of city branding. Routledge.

Beckouch, P. (2019). Les nouveaux territoires du numérique : l'univers digital du sur-mesure de masse. Éditions Sciences humaines.

Ben Hadj Mohamed, A. (2015). Réseau de capteurs sans fil comportementaux pour l'aide au maintien à domicile par la surveillance en babitat intelligent [Thèse de doctorat, Université de Toulouse]. Archive ouverte HAL. https://tel.archives-ouvertes.fr/tel-01330973/document

Bertuglia, C. (2020). Reconnaissance d'activités dans un habitat intelligent à l'aide de radars Ultra-Wideband [Mémoire de maitrise, Université du Québec à Chicoutimi]. Constellation. https://constellation.uqac.ca/5917/1/Bertuglia_uqac_0862_10706.pdf

Boutros, M. (2019, 28 octobre). La ville intelligente, levier d’une révolution sociale et écologique. Le Devoir. https://www.ledevoir.com/societe/transports-urbanisme/565742/urbanisme-la-ville-intelligente-levier-d-une-revolution-socialeet-ecologique

Breux, S. et Diaz, J. (2017). La ville intelligente : origine, défnitions, forces et limites d'une expression polysémique [Rapport de recherche]. Centre Urbanisation Culture Société de l'INRS. http://espace.inrs.ca/id/eprint/4917/1/Rapport-LaVilleIntelligente.pdf

Briner, C. (2020, 15 mars). La Chine veut noter tous ses habitants et installe 600 millions de caméras. Radio Télévision Suisse. https://www.rts.ch/info/monde/11137943-la-chine-veut-noter-tous-ses-habitants-et-installe-600-millions-de-cameras.html

Cattaruzza, A. (2019). Géopolitique des données numériques : pouvoir et conflits à l'heure du Big Data. Éditions Le Cavalier bleu.

Courmont, A. et Le Galès, P. (dir.). (2019). Gouverner la ville numérique. PUF. 
Courmont, A. et Vincent, M. (2020, 25 juin). Smart cities : des politiques numériques faiblement politisées? Métropolitiques. https://metropolitiques.eu/Smart-Cities-des-politiques-numeriques-faiblement-politisees.html

Damon, J. (2016). La Smart Cité en 2040 : une utopie urbaine en deux fictions. Annales des mines, 84(4), 5-9. https://www.cairn.info/revue-responsabilite-et-environnement-2016-4-page-5.htm

Dirks, S. et Keeling, M. (2009). A vision of smarter city: How cities can lead the way into a prosperous and sustainable future. IBM Institute for Business Value. https://www.ibm.com/downloads/cas/2JYLM4ZA

Durand Folco, J. (2019). La «ville intelligente», au service de qui? Relations, 804, 29-30. https://id.erudit.org/iderudit/91735ac

Eveno, E. (2018). La ville intelligente : objet au cœur de nombreuses controverses. Quaderni, 96, 29-41. http://journals.openedition.org/quaderni/1174

Fonds des Nations unies pour la population (UNFPA). (2007) État de la population mondiale 2007. https://ise.unige.ch/isdd/IMG/pdf/pop_mondiale_2007.pdf

Gagné, J.-F. (2019). La ville intelligente : défis pour la démocratie. Dans E. Caccamo, J. Walzberg, T. Reigeluth et N. Merveille (dir.), De la ville intelligente à la ville intelligible (p. 15-32). PUQ.

Gassmann, O., Böhm, J. et Palmié, M. (2019). Smart cities: Introducing digital innovation to cities. Emerald.

Goin, J.-B. (2017, 13 juin). La ville intelligente. Villes Régions Monde. http://www.vrm.ca/la-ville-intelligente

Haëntjens, J. (2018). Comment les géants du numérique veulent gouverner nos villes : la cité face aux algorithmes. Éditions Rue de l'Échiquier.

Halegoua, G. R. (2020). Smart cities. MIT Press.

Halin, F. (2017, 16 juillet). Le Québec est un paradis des centres de données. La Presse. https://www.journaldemontreal.com/2017/07/16/le-quebec-est-un-paradis--des-centres-de-donnees

Harari, Y. N. (2018). 21 leçons pour le XXIe siècle. Albin Michel.

Henriot, C. (2018). La politique de la ville intelligente en Chine : ancrage local d'un modèle urbain globalisé. Flux, 114(4), 71-85. https://doi.org/10.3917/flux1.114.0071

Joarson. (2021, 18 mars). Data center : l'impact des infrastructures sur l'environnement et les solutions possibles. Lebigdata.fr. https://www.lebigdata.fr/data-center-impact-environnement

Katundi, E. (2018). La coévolution des villes intelligentes et l'internet des objets : le cas de la ville de Montréal [Mémoire de maitrise, Université du Québec à Montréal]. Archipel. https://archipel.uqam.ca/11835/1/M15653.pdf

Khedira, H. (2020). Smart city : une mise au pas des villes par l'innovation technologique. Dans M. Adam et É. Comby (dir.), Le capital dans la cité : une encyclopédie critique de la ville (p. 337-349). Éditions Amsterdam.

Languillon-Aussel, R., Leprêtre, N. et Granier, B. (2016). La stratégie de la «smart city» au Japon : expérimentations nationales et circulations globales. EchoGéo, 36. https://doi.org/10.4000/echogeo.14598

Lévesque, S. (2019). Don de soi et servitude volontaire. Dans E. Caccamo, J. Walzberg, T. Reigeluth et N. Merveille (dir.), De la ville intelligente à la ville intelligible (p. 55-72). PUQ.

McKenna, A. (2021, 2 juin). Des feux de circulation intelligents pour réduire les émissions de GES. Le Devoir. https://www.ledevoir.com/economie/606489/environnement-des-feux-de-circulation-intelligents-pour-reduire-les-emissions-deges\#: : :text $=$ Ce $\% 20$ syst $\%$ C3 $\%$ A 8 me $\% 20$ ne $\% 20$ recourt $\% 20 \%$ C3 $\%$ A0,dehors $\% 20$ des $\% 20$ heures $\% 20$ de $\% 20$ pointe

Meijer, A. et Rodríguez Bolívar, M. (2016). La gouvernance des villes intelligentes : analyse de la littérature sur la gouvernance urbaine intelligente. Revue internationale des sciences administratives, 2(2), 417-435. https://doi.org/10.3917/risa.822.0417

Merlet, L. (2018). Habitat intelligent: jusqu'où l'habitant est-il maître des lieux? [Mémoire de maîtrise, École nationale supérieure d'architecture de Nantes]. Dumas. https://dumas.ccsd.cnrs.fr/dumas-01996256

Morozov, E. et Bria, F. (2018) Rethinking smart city: Democratizing urban technology. Rosa Luxemburg Stiftung. https:// rosalux.nyc/rethinking-the-smart-city-democratizing-urban-technology

Mosco, V. (2019). The smart city in a digital world. Emerald. 
Paquot, Th. (2020). Mesure et démesure des villes. CNRS.

Pelissier, M. et Pybourdin, I. (2009). L'intelligence territoriale: Entre structuration de réseau et dynamique de communication. Les Cabiers du numérique, 5, 93-109. https://doi.org/

Perreault, M. (2019, 25 août). Les centres de données en ébullition. La Presse. https://www.lapresse.ca/actualites/sciences/ 2019-08-25/les-centres-de-donnees-en-ebullition

Proulx, B. (2021, 6 août). Ces algorithmes qui nous divisent et nous polarisent. Le Devoir. https:/ /www.ledevoir.com/ societe/600191/serie-tous-polarises-ces-algorithmes-qui-nous-divisent

Quesnot, T. (2016). L’involution géographique : des données géosociales aux algorithmes. Netcom, 30(3/4), 281-304. http://journals.openedition.org/netcom/2545

Shearmur, R., Charron, M. et Pajevic, F. (2020). Pourquoi seules les villes sont-elles qualifiées d'intelligentes? Un vocabulaire du biais urbain. Le Géographe canadien, 64(22), 310-322. https://doi.org/10.1111/cag.12573

Theys, J. (2020). Le développement durable face à sa crise : un concept menacé, sous-exploité ou dépassé? Développement durable et territoires, 11(2). http://journals.openedition.org/developpementdurable/17468

Tremblay, D.-G. et Tremblay, R. (dir.). (2006). La compétitivité urbaine à l'ère de la nouvelle économie : enjeux et défis. PUQ. https://extranet.puq.ca/media/produits/documents/818_9782760521742.pdf

Tribillon, J.-F. (2009) L'urbanisme. La Découverte.

Vaccari, V. (1957). Progrès technique et progrès social. Relations industrielles, 12(4), 326-347. https://doi.org/10.7202/1022510ar

Vidal, P. et Gauthier, M.-H. (2021). La prise en compte du numérique par les agences d'urbanisme : évaluation de la situation française. Cybergeo, 980. https://doi.org/10.4000/cybergeo.36839

Zuboff. S. (2019). L'âge du capitalisme de surveillance. Zulma. 